\title{
Colloidal $\mathrm{CuFeS}_{2}$ Nanocrystals: Intermediate Fe d-Band Leads to High Photothermal Conversion Efficiency
}

Sandeep Ghosh, ${ }^{*}, \dagger, \square$ Tommaso Avellini, ${ }^{\perp,} \square$ Alessia Petrelli, ${ }^{\perp,} \square$ Ilka Kriegel, ${ }^{\dagger}, \square$ Roberto Gaspari, ${ }^{\S,}, \square$ Guilherme Almeida, ${ }^{\dagger}, \#$ Giovanni Bertoni, ${ }^{\dagger, \text { II }}$ Andrea Cavalli, ${ }^{\S}, \square$ Francesco Scotognella, ${ }^{\|}$ Teresa Pellegrino, ${ }^{*, \perp}$ and Liberato Manna*, ${ }^{\dagger}$

${ }^{\dagger}$ Department of Nanochemistry, ${ }^{\S}$ CompuNet, and ${ }^{\perp}$ Department of Drug Discovery and Development, Istituto Italiano di Tecnologia (IIT), via Morego 30, 16163 Genova, Italy

IIMEM-CNR, Parco Area delle Scienze 37/A, 43124 Parma, Italy

\#Dipartimento di Chimica e Chimica Industriale, Università degli Studi di Genova, Via Dodecaneso, 31, 16146 Genova, Italy

"Dipartimento di Fisica, Politecnico di Milano, Piazza Leonardo da Vinci 32, 20133 Milano, Italy

\section{Supporting Information}

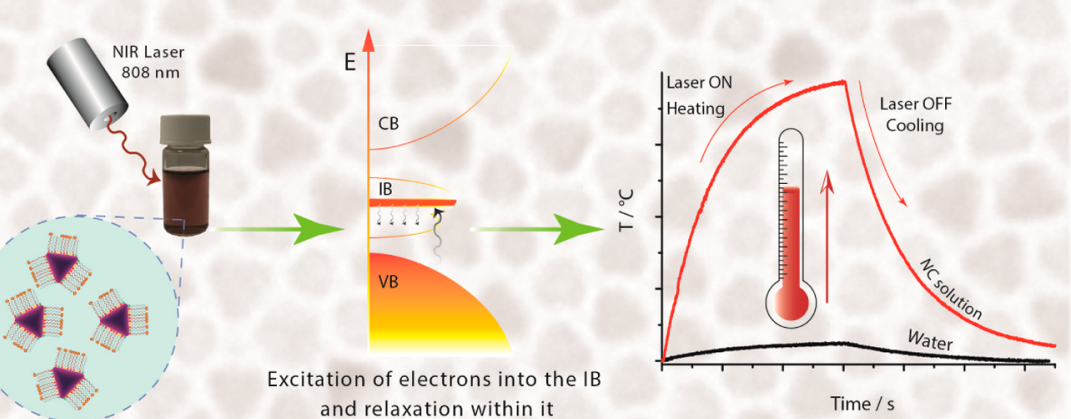

ABSTRACT: We describe the colloidal hot-injection synthesis of phase-pure nanocrystals (NCs) of a highly abundant mineral, chalcopyrite $\left(\mathrm{CuFeS}_{2}\right)$. Absorption bands centered at around 480 and $950 \mathrm{~nm}$, spanning almost the entire visible and nearinfrared regions, encompass their optical extinction characteristics. These peaks are ascribable to electronic transitions from the valence band (VB) to the empty intermediate band (IB), located in the fundamental gap and mainly composed of Fe $3 \mathrm{~d}$ orbitals. Laser-irradiation (at $808 \mathrm{~nm}$ ) of an aqueous suspension of $\mathrm{CuFeS}_{2}$ NCs exhibited significant heating, with a photothermal conversion efficiency of $49 \%$. Such efficient heating is ascribable to the carrier relaxation within the broad IB band (owing to the indirect VB-IB gap), as corroborated by transient absorption measurements. The intense absorption and high photothermal transduction efficiency (PTE) of these NCs in the so-called biological window (650-900 nm) make them suitable for photothermal therapy as demonstrated by tumor cell annihilation upon laser irradiation. The otherwise harmless nature of these NCs in dark conditions was confirmed by in vitro toxicity tests on two different cell lines. The presence of the deep Fe levels constituting the IB is the origin of such enhanced PTE, which can be used to design other high performing NC photothermal agents.

\section{INTRODUCTION}

Chalcopyrite $\left(\mathrm{CuFeS}_{2}\right)$, a mineral with golden luster, is an $\mathrm{n}$ type semiconductor that exhibits large thermoelectric power, ${ }^{1}$ rectification, ${ }^{2}$ antiferromagnetism with Néel temperature of 823 $\mathrm{K},{ }^{1}$ and an unusually low optical band gap of $0.53 \mathrm{eV}^{3}$ Chalcopyrite crystallizes in a substitutional analogous structure to that of carbon, which means that all the atoms are tetrahedrally coordinated in the lattice, belonging to the tetragonal space group $\mathrm{I} \overline{4} 2 \mathrm{~d} .{ }^{4}$ Despite being composed of earth abundant elements like $\mathrm{Cu}$ and $\mathrm{Fe}$, there are very few comprehensive studies on nanocrystals (NCs) of this Cu-based semiconductor in the literature. ${ }^{5,6}$ Silvester et al. have reported the hydrothermal synthesis of optically transparent colloidal sols of chalcopyrite NCs through the controlled conversion of amorphous iron(III) oxide particles, ${ }^{7}$ with optical characteristics fairly matching to those of bulk. ${ }^{8}$ Wang et al. ${ }^{9}$ and Liang et al. $^{10}$ have described colloidal approaches to synthesize $\mathrm{CuFeS}_{2} \mathrm{NCs}$, although the optical properties and electronic band structure have not been explored in much detail. For instance, Liang et al. reported a band gap of $1.2 \mathrm{eV}$ for the $\mathrm{CuFeS}_{2} \mathrm{NCs}$, which is reminiscent of the plasmonic absorption

Received: May 31, 2016

Revised: June 1, 2016

Published: June 2, 2016 
of $\mathrm{Cu}_{2-x} \mathrm{~S} \mathrm{NCs}{ }^{11}$ suggesting that their samples probably contained a fraction of $\mathrm{Cu}_{2-x} \mathrm{~S}$ NCs.

Here, we report a synthesis protocol that delivers nearly monodisperse $\mathrm{CuFeS}_{2} \mathrm{NCs}$ that are free from contaminant phases since to date there have been no consistent reports on phase pure, stable, and monodisperse NCs for this material. The optical reflectivity spectrum of a single crystal of $\mathrm{CuFeS}_{2}$ is characterized by strong absorption bands at around 1.0 and 2.1 $\mathrm{eV}$ and an absorption edge at around $3.2 \mathrm{eV},{ }^{8}$ as also present in the optical absorption spectra from solutions of the NCs prepared by us. These absorption characteristics arise from an intermediate band (IB) in the fundamental gap, formed predominantly by empty $\mathrm{Fe} 3 \mathrm{~d}$ orbitals. Electronic transitions to the IB from the valence band (VB) would explain the lowerenergy absorption bands, while the edge at $3.2 \mathrm{eV}$ can be assigned to the VB to conduction band (CB) transitions. ${ }^{12-14}$ The top of the VB and the bottom of the IB are found to be separated by an indirect gap of $0.53 \mathrm{eV}^{3}$

Iron is known to introduce deep intermediate trap states in the fundamental gap of most photovoltaic semiconductors and hence is meticulously avoided in commercial silicon photovoltaics since it drastically affects the solar cell efficiency by introducing numerous recombination centers. ${ }^{15}$ In $\mathrm{CuFeS}_{2}$, iron can be considered to play a very similar role since the broad IB is mainly composed of Fe $3 \mathrm{~d}$ orbitals and is generally absent in other chalcopyrite-type semiconductors like $\mathrm{CuGaS}_{2}$ and $\mathrm{CuAlS}_{2}{ }^{16}$ This band structure is indeed supported by density functional theory (DFT) calculations performed by us and reported here, which yield an indirect VB-IB gap of 0.63 $\mathrm{eV}$, in reasonable agreement with the bulk experimental value. The presence of an indirect $\mathrm{VB}-\mathrm{IB}$ gap means that the electrons photoexcited to the IB will relax through thermal nonemissive pathways. Indeed, transient absorption (TA) measurements on a solution of the NCs synthesized by us are consistent with thermalization of the photoexcited carriers within the broad IB, followed by the interband relaxation from the IB to the VB (slower).

Here, we take advantage of this unique band structure to demonstrate that $\mathrm{CuFeS}_{2}$ NCs can be employed as efficient light-to-heat converters in the biological window, owing to their low optical gap. Likewise, the as-synthesized NCs, coated with a commercially available amphiphilic polymer that made them dispersible in aqueous solutions, exhibited significant heating in water upon exposure to a laser at $808 \mathrm{~nm}$, with photothermal transduction efficiency (PTE) of $49 \%$. This notion is further corroborated by the high molar attenuation coefficient $(\varepsilon=5.2$ $\times 10^{6} \mathrm{M}^{-1} \mathrm{~cm}^{-1}$ ) of the NCs at this irradiation wavelength. We further demonstrate that these characteristics make the NCs of chalcopyrite $\left(\mathrm{CuFeS}_{2}\right)$ efficient agents for photothermal tumor therapy. Laser-induced ablation of cancer cells or photothermal therapy (PT) has been gaining increasing importance in recent years as a viable method of cancer treatment, ${ }^{17}$ mostly due to its much minimally invasive nature. ${ }^{18}$ To be fit for PT applications, the sensitizers must: (i) exhibit strong absorption in the near-infrared (NIR) region, especially in the 650-900 $\mathrm{nm}$ range, (where light extinction by hemoglobin and water is minimal) ${ }^{19}$ and (ii) efficiently convert the absorbed NIR radiation into heat. In metallic or heavily doped systems with a high density of free carriers, like plasmonic nanomaterials of $\mathrm{Au}^{20-23}$ and copper chalcogenides, ${ }^{24-28}$ heat release occurs due to the excitation of localized surface plasmon resonances. Immediately after excitation, the hot carriers thermalize and cool through the emission of phonons by interacting with the crystal lattice of the nanoparticle. This leads to heating of the nanoparticle, which is then exploited for PT. Heat generation is limited to the region of the plasmon resonance, which itself depends on the carrier density, the size, and the shape of the nanoparticle. ${ }^{29}$ Recently, various semiconductor NCs, among which $\mathrm{Ge}^{30}{ }^{30} \mathrm{Si}^{31} \mathrm{WS}_{2},{ }^{32,33} \mathrm{~W}_{18} \mathrm{O}_{49}{ }^{34} \mathrm{Bi}_{2} \mathrm{~S}_{3},{ }^{35} \mathrm{Nd} \mathrm{LaF}_{3},{ }^{36}$ and $\mathrm{NdVO}_{4}{ }^{37}$ have also been explored as PT agents, although in most of these reports, the mechanism and the essential requirements for light-to-heat conversion have not been thoroughly discussed. For semiconductors in general, carriers with excess energy (above the band edge) relax by emission of phonons, which ultimately leads to heating of the material. ${ }^{38}$ For photothermal applications in the NIR, this is however limited to semiconductors with a sufficiently low band gap.

The $\mathrm{CuFeS}_{2} \mathrm{NCs}$ synthesized by us fulfill both the criteria of efficient NIR light to heat conversion and strong absorption in the NIR region. However, it was necessary to demonstrate the therapeutic ability of these NCs on live cells upon laser irradiation and check for their cytotoxicity under radiationless conditions. An ideal photothermal agent should not be toxic to live cells in dark conditions. To this effect, viability tests on two different cell lines were undertaken to ascertain the cytotoxicity of $\mathrm{CuFeS}_{2} \mathrm{NCs}$ under radiationless conditions. The cells could tolerate a NC dose of up to $50 \mathrm{ppm}$ of copper content for $24 \mathrm{~h}$ in the dark. However, upon prolonging the exposure to $72 \mathrm{~h}$ in the dark, even lower NC doses (less than $10 \mathrm{ppm}$ in $\mathrm{Cu}$ ) were found to be cytotoxic. The therapeutic ability of the NCs was then demonstrated through annihilation of the NC-imbibedcells under laser irradiation ( $808 \mathrm{~nm}$, in the biological window), even at NC doses far lower than the toxic limit. A NC dose of around $3 \mathrm{ppm}$ (in $\mathrm{Cu}$ ) was identified as the therapeutic limit, i.e., this dose is efficient in annihilating cells under laser irradiation but otherwise harmless in the dark. The efficient light to heat conversion (PTE: 49\%), strong NIR absorption $(\varepsilon$ $=5.2 \times 10^{6} \mathrm{M}^{-1} \mathrm{~cm}^{-1}$ at $\left.808 \mathrm{~nm}\right)$, and small size $(21 \mathrm{~nm}$ hydrodynamic diameter, which would ensure longer bloodstream circulation time) demonstrate that chalcopyrite NCs can be employed as photothermal agents.

\section{EXPERIMENTAL SECTION}

Materials. Copper(I) iodide (CuI, $\geq 99.5 \%$ ), iron(III) acetylacetonate $\left(\mathrm{Fe}(\mathrm{acac})_{3}, \geq 99.9 \%\right)$, 1-octadecene (ODE, technical grade, $90 \%$ ), oleylamine (OLAM, technical grade, 70\%), and 1-dodecanethiol (DT, $\geq 98 \%)$ were purchased from Sigma-Aldrich. Tri-n-octylphosphine (TOP, 97\%) was procured from Strem Chemicals. $\alpha$-Methoxy$\omega$-mercapto polyethylene glycol (SH-PEG-OCH 3 MW: $2000 \mathrm{~g} \mathrm{~mol}^{-1}$ ) was purchased from Rapp Polymere. Anhydrous chloroform, methanol, and isopropanol were purchased from Carlo Erba reagents. All chemicals were used as received. A Milli-Q Millipore system was used for the purification of water (resistivity $\geq 18 \mathrm{M} \Omega \mathrm{cm}$ ).

Synthesis of $\mathrm{CuFeS}_{2} \mathrm{NCs}$. All synthesis procedures were undertaken by employing standard Schlenk line techniques assisted by a nitrogen-filled glovebox. Typically, a mixture of CuI (190 mg, 1 $\mathrm{mmol}$ ) and TOP (380 $\mathrm{mg}$ (amount adjusted as per chemical assay), 1 $\mathrm{mmol}$ ) in a 1:1 molar ratio was prepared in ODE $(1 \mathrm{~mL})$. The mixture was homogenized, aided by mild heating and stirring, until all the $\mathrm{CuI}$ dissolved resulting in a transparent solution. Care has to be exercised as to prepare this solution in an inert atmosphere. This solution was then transferred to a mixture of $\mathrm{Fe}(\mathrm{acac})_{3}(353 \mathrm{mg}, 1 \mathrm{mmol})$ and ODE $(6 \mathrm{~mL})$ kept in a $25 \mathrm{~mL}$ round-bottomed flask. The final mixture was then subjected to evacuation at $80{ }^{\circ} \mathrm{C}$ for a period of $1 \mathrm{~h}$ to let the mixture dry. Thereafter, the flask was filled with nitrogen, and the temperature was raised to $280^{\circ} \mathrm{C}$. A solution of $1 \mathrm{~mL}$ of DT and $2 \mathrm{~mL}$ of OLAM, separately prepared by degassing the mixture at $80^{\circ} \mathrm{C}$ and subsequently heating it under nitrogen flow to $160^{\circ} \mathrm{C}$, was injected at 
this point. The temperature dropped to $260{ }^{\circ} \mathrm{C}$ and was allowed to recover to $270{ }^{\circ} \mathrm{C}$. The initial dark red solution (color ascribed to $\left.\mathrm{Fe}(\mathrm{acac})_{3}\right)$ turned dark brown within the first few minutes, which signified the formation of the $\mathrm{CuFeS}_{2} \mathrm{NCs}$. The reaction mixture was allowed to stir for $15 \mathrm{~min}$ at this temperature, and finally the heating mantle was removed to cool the reaction mixture. The final dark purple solution was then washed with chloroform/methanol mixture. The NCs were resuspended in chloroform and subjected to a further centrifugation at $1000-2000 \mathrm{rpm}$ for $1-2 \mathrm{~min}$ to remove larger particles formed during the reaction.

X-ray Diffraction (XRD) Analysis. Concentrated solutions of the NC samples were drop-cast (followed by evaporation of the solvent) on a zero background silicon substrate to acquire the XRD patterns, which were performed on a Rigaku SmartLab $9 \mathrm{~kW}$ diffractometer with the X-ray source operating at $40 \mathrm{kV}$ and $150 \mathrm{~mA}$. The instrument was equipped with a $\mathrm{Cu}$ source and a Göbel mirror (to obtain a parallel beam and suppress the $\mathrm{Cu} K \beta$ radiation at $1.392 \AA$ ) and was used in the $2 \theta / \omega$ scan geometry for data acquisition. Phase identification was performed through the assistance of PDXL software.

Steady State UV-Vis-NIR Extinction Spectroscopy. Optical extinction spectra of dilute chloroform dispersions of the NC samples were recorded in quartz cuvettes of $1 \mathrm{~cm}$ path-length employing a Varian Cary 5000 UV-Vis-NIR absorption spectrophotometer in the wavelength range of $300-2200 \mathrm{~nm}$.

Elemental Analysis. The composition and solution concentration of the NCs were ascertained using inductively coupled plasma-optical emission spectroscopy (ICP-OES) analysis performed on aiCAP 6000 spectrometer (ThermoScientific). The samples were digested overnight in aqua regia and diluted to a known amount before the measurements.

Dynamic Light Scattering (DLS) Measurements. The hydrodynamic diameter of the NCs was determined by DLS measurements on a Malvern Zetasizer (Nano Series, Nano ZS) instrument. The scattered intensity was collected at $173^{\circ}$ back scattered geometry with a $632 \mathrm{~nm}$ laser source. For each sample, three measurements were taken with 10-20 acquisitions in each case.

Transmission Electron Microscopy (TEM) Analysis. Brightfield (BF)TEM images and selected area electron diffraction (SAED) patterns were acquired on samples prepared by drop-casting a concentrated solution of NCs on carbon-coated 200 mesh copper grids using a JEOL JEM-1011 microscope (W filament) operated at $100 \mathrm{kV}$ accelerating voltage. High-resolution (HR)TEM, high-angle annular dark-field (HAADF), and energy dispersive $\mathrm{X}$-ray spectroscopy (EDS) analyses were performed on a JEOL JEM-2200FS microscope equipped with a Schottky emitter at $200 \mathrm{kV}$, a CEOS image corrector allowing for an information limit of $0.8 \AA$, and an incolumn energy filter ( $\Omega$-type). The chemical compositions of the NCs were determined by EDS using a JEOL JED-2300 Si $(\mathrm{Li})$ detector. The $\mathrm{NC}$ suspensions were deposited onto ultrathin carbon coated Au grids, and the measurements were carried out using a holder with a beryllium cup for background reduction in the spectra.

Computational Details. Calculations have been performed considering the orthorhombic unit cell, which is composed of $4 \mathrm{Fe}$, $4 \mathrm{Cu}$, and $8 \mathrm{~S}$ atoms, using the plane wave code pwscf. ${ }^{39}$ For all calculations, we employed ultrasoft pseudopotentials, a Brillouin zone sampling using a $4 \times 4 \times 4$ Monkhorst-Pack mesh, ${ }^{40}$ and a plane wave cutoff of 30 and $240 \mathrm{Ry}$ for the wave function and charge density expansion, respectively. The PBE functional ${ }^{41}$ and linear response $\mathrm{DFT}+\mathrm{U}^{42}$ was used. The system geometry was relaxed until the largest force on atoms was smaller than $10^{-4} \mathrm{Ry} / \mathrm{Bohr}$ and the largest stress tensor component was smaller than $3 \times 10^{-5} \mathrm{Ry} / \mathrm{Bohr}^{3}$. Spin polarization was allowed to reproduce the antiferromagnetic ground state of the system (see also Figure S3). Further details can be found in the Supporting Information.

Transient Absorption (TA) Spectroscopy. Ultrafast pumpprobe measurements were performed on a $\mathrm{Ti}$ /sapphire chirp pulse amplified source, with maximum output energy of about $1 \mathrm{~mJ}, 1 \mathrm{kHz}$ repetition rate, central wavelength of $800 \mathrm{~nm}$, and pulse duration of about 150 fs. Pump pulses of $490 \mathrm{~nm}$ were generated by optical parametric amplification (OPA) in a $\beta$-Barium borate $(\mathrm{BBO})$ crystal.
White light was generated in a thin sapphire plate in approximately the range of $430-700 \mathrm{~nm}$. The detection system was based on a fast optical multichannel analyzer (OMA) with dechirping algorithm to obtain chirp-free transient transmission spectra measured as the normalized transmission change, $\Delta T / T$. The excitation energy per pulse was kept in the linear regime with a maximum fluence of $\sim 80$ $\mu \mathrm{J} / \mathrm{cm}^{2}$. All measurements were performed at room temperature on sealed samples prepared under nitrogen atmosphere by dispersing the $\mathrm{CuFeS}_{2} \mathrm{NCs}$ in chloroform.

Ligand Exchange and Water Transfer. The $\mathrm{CuFeS}_{2} \mathrm{NCs}$ were transferred to water by replacing the native ligands with hydrophilic thiol terminated polyethylene glycol (PEG) molecules, SH-PEG$\mathrm{OCH}_{3}$. In a typical exchange procedure, $3 \mathrm{~mL}$ of the $\mathrm{NC}$-solution in chloroform $(3.0 \mu \mathrm{M}, 12.6 \mathrm{~nm}$ tetrahedral edge) was mixed with a solution of SH-PEG-OCH $\mathrm{OCH}_{3}$ in methanol $(248 \mathrm{mg}$ dissolved in $15 \mathrm{~mL}$ of methanol). The amount of PEG units was roughly determined based on a fixed number of ligand molecules per unit NC surface area (50 ligand molecules per square $\mathrm{nm}$ of $\mathrm{NC}$ surface, in this case). The mixture was shaken vigorously for $2 \mathrm{~h}$, and the PEG-coated NCs were then washed with $20 \mathrm{~mL}$ of hexane. The concentrated NC solution was diluted with about $4 \mathrm{~mL}$ of methanol, and the washing step described earlier was repeated twice to ensure an efficient removal of the hydrophobic ligands (e.g., dodecanethiol/oleylamine). The solvent from the PEG-coated NC solution was then removed under reduced pressure yielding the dried NCs, which produced a clear homogeneous purple solution when dispersed in water. Some large NC aggregates were formed during these manipulations and were removed by syringe filtration $(0.2 \mu \mathrm{m}$ pore size $)$. Finally, the excess of free PEG polymer was removed with two cycles of water dilution/concentration filtration using a Millipore Amicon centrifuge filter (100 kDa MWCO, 2300 rpm, $10 \mathrm{~min}$ ).

PTE Calculation. The PTE of the PEG-coated NCs was determined according to the protocol of Roper et al. ${ }^{43} \mathrm{~A}$ quartz cuvette, which contained an aqueous solution of NCs, was placed inside a vacuum chamber and irradiated with a $808 \mathrm{~nm}$ continuous laser (RTLMDL-808-5W, Roithner Laser Technik) while the temperature was monitored using a thermo-probe (FOT Lab Kit equipped with Fluoroptic probe, Luxtron). The laser was switched off when the solution temperature reached a plateau and the temperature versus time profile of the solution was recorded. As a control, the same heating-cooling cycle was performed for a quartz cuvette filled with the same volume of deionized water. From the cooling profiles, the PTE was determined (see Section S4 of the Supporting Information for details).

Cell Culture. Two cell lines were employed in our experiments. Human epithelial carcinoma cells (HeLa, ATCC, UK) were cultured in Dulbecco's modified Eagle medium (DMEM, Gibco, UK), and human ovarian cancer cells (IGROV 1, ATCC, UK) were cultured in Roswell Park Memorial Institute medium (RPMI, Gibco,UK) both supplemented with $10 \%$ inactivated fetal bovine serum (FBS), $1 \%$ penicillin streptomycin (PS), and $1 \%$ glutamine at $37{ }^{\circ} \mathrm{C}$, in $95 \%$ humidity and $5 \% \mathrm{CO}_{2}$. Cells were split every 3-4 days before they reached $80 \%$ confluence.

Stability of PEG-Coated NCs in Physiological Conditions. NCs at a dose of $10 \mathrm{ppm}$ of $\mathrm{Cu}$ were dispersed in complete DMEM medium and kept at $37^{\circ} \mathrm{C}$, at $95 \%$ humidity and $5 \% \mathrm{CO}_{2}$, for different intervals of time. The hydrodynamic diameter was monitored over time for the first $24 \mathrm{~h}$ by DLS.

Cell Viability Tests. The NC doses to add to the cell dish were standardized against the copper content of the NCs. The PrestoBlue (PB) assay was performed according to the manufacturer's protocol (Invitrogen, Carlsbad, CA, USA). Briefly, $10^{4} \mathrm{HeLa}$ or IGROV 1 cells/ well were seeded in a 96 multiwell plate and allowed to adhere to the well dish. After $24 \mathrm{~h}$ of incubation, NCs at increasing doses (measured as the $\mathrm{Cu}$ concentration, $[\mathrm{Cu}]$, ranging from $1-50 \mathrm{ppm}$ ) were then added to the media and incubated for additional 24 and $72 \mathrm{~h}$ at $37^{\circ} \mathrm{C}$. The PB reagent was then added directly to the cells in the culture medium and incubated for additional $60 \mathrm{~min}$ at $37{ }^{\circ} \mathrm{C}$. The cell viability was determined at this stage by recording the absorbance for each well at 570 and $600 \mathrm{~nm}$. The absorbance ratios (A570/A600 nm) 
for each well were normalized with respect to the absorbance ratio of control cells wells, which were not treated with $\mathrm{CuFeS}_{2} \mathrm{NCs}$.

In Vitro Photothermal Ablation Experiments. HeLa cells (2 $\times 10^{5}$ cells/well) were seeded in a six-well plate and allowed to adhere for $24 \mathrm{~h}$. NCs (at $\mathrm{Cu}$ content ranging from 3-20 ppm) were then added to the cell media and incubated for an additional $24 \mathrm{~h}$ at $37^{\circ} \mathrm{C}$. The cells were then rinsed with phosphate-buffered saline (PBS) to remove the noninternalized NCs and collected by centrifugation after being detached by trypsin treatment. The cells were then transferred to a $2 \mathrm{~mL}$ Eppendorf tube so that $10^{6}$ cells were present in $0.5 \mathrm{~mL}$ of complete DMEM media so that they were ready for the irradiation experiments. Irradiation experiments were performed using an $808 \mathrm{~nm}$ continuous laser set-up (RLTMDL-808-5W, Roithner Laser Technik, power adjusted to $1.14 \mathrm{~W}$, spot size $0.36 \mathrm{~cm}^{2}$, and power density to 3.1 $\mathrm{W} \mathrm{cm}{ }^{-2}$ ) equipped with a controlled temperature chamber to fix the temperature of the sample holder at $37.0 \pm 0.1{ }^{\circ} \mathrm{C}$. The irradiation time for each experiment was set at $13 \mathrm{~min}$, and the temperature was monitored during the treatment using a thermo-camera (OPTRIS, PI230). Temperature profiles were recorded on cell suspensions and also on solutions containing only NCs (for comparison) at NC concentrations corresponding to those administrated to the cells. To this aim, NC solutions containing 3, 5, 10, $20 \mathrm{ppm}$ of $\mathrm{Cu}$, respectively, were dispersed in a final volume of $0.5 \mathrm{~mL}$ of complete media (DMEM) and irradiated at $808 \mathrm{~nm}\left(1.14 \mathrm{~W}, 3.1 \mathrm{~W} \mathrm{~cm}^{-2}\right)$ for $13 \mathrm{~min}$. To test the toxic effects of the irradiation on the cells, the radiation treated HeLa cells were reseeded in a 24-well plate at a density of $2 \times$ $10^{5}$ cells/well, and their viability was analyzed by PB assay after 24 and $72 \mathrm{~h}$ of incubation at $37{ }^{\circ} \mathrm{C}$. The cell viability was also assessed through fluorescence imaging. The LIVE/DEAD viability assay was performed according to the manufacturer's protocol (Thermo Fisher Scientific). Briefly, after NIR irradiation experiments, $2 \times 10^{5} \mathrm{HeLa}$ cells/well were seeded in a six-well plate and allowed to adhere for $5 \mathrm{~h}$, after which they were washed gently in PBS and incubated in dark conditions for 45 min with $100 \mu \mathrm{L}$ per well of LIVE/DEAD reagents. The LIVE/DEAD reagent is composed of $20 \mu \mathrm{L}$ of the supplied $2 \mathrm{mM}$ ethidium homodimer (EthD-1) stock solution and $5 \mu \mathrm{L}$ of $4 \mathrm{mM}$ calcein AM dye of the supplied stock solution in $10 \mathrm{~mL}$ of sterile PBS. Green fluorescence for calcein AM, corresponding to living cells, and red fluorescence for EthD-1, corresponding to dead cells, were detected using a Nikon A1 confocal microscope.

\section{RESULTS AND DISCUSSION}

Synthesis and Characterization of the NCs. In our synthesis, we prepared phase pure NCs through a colloidal approach wherein copper(I) iodide (CuI) and iron(III) acetylacetonate were employed as the respective $\mathrm{Cu}$ and $\mathrm{Fe}$ precursors and 1-dodecanethiol (DT) as the S-source, which was delivered via hot-injection, in the presence of oleylamine as a capping agent. $\mathrm{CuI}$ was first dissolved in TOP in the presence of ODE since it is otherwise insoluble in ODE and also since TOP is known to favor the formation of the ternary chalcopyrite phase. ${ }^{44} \mathrm{CuI}$ was chosen as the $\mathrm{Cu}$-precursor since other copper halides like $\mathrm{CuCl}$ and $\mathrm{CuBr}$ led to the formation of $\mathrm{Cu}-\mathrm{S}$ impurity phases. This happens due to the increased reactivity of $\mathrm{Cu}^{+}$ions (soft acid) toward the thiol moiety (soft base) when present in association with harder bases like $\mathrm{Cl}^{-}$or $\mathrm{Br}^{-}$. $\mathrm{I}^{-}$being a much softer base reduces the rate of $\mathrm{Cu}^{+}$reaction with DT and balances the reactivity of the two metal precursors, which leads to phase purity. ${ }^{45}$ The reaction led to dark purple colloidal dispersions of chalcopyrite NCs, as shown in Figure 1, panel a. The phase purity of the assynthesized $\mathrm{CuFeS}_{2}$ NCs was ascertained through XRD and azimuthally integrated SAED patterns, as shown in Figure 1, panel $b$, which confirmed the tetragonal (I $\overline{4} 2 \mathrm{~d})$ crystal structure as reported for the bulk structure by Knight et al. ${ }^{4}$

The chalcopyrite phase was discernible within the first few minutes after the injection of the sulfur source (DT-OLAM (a)
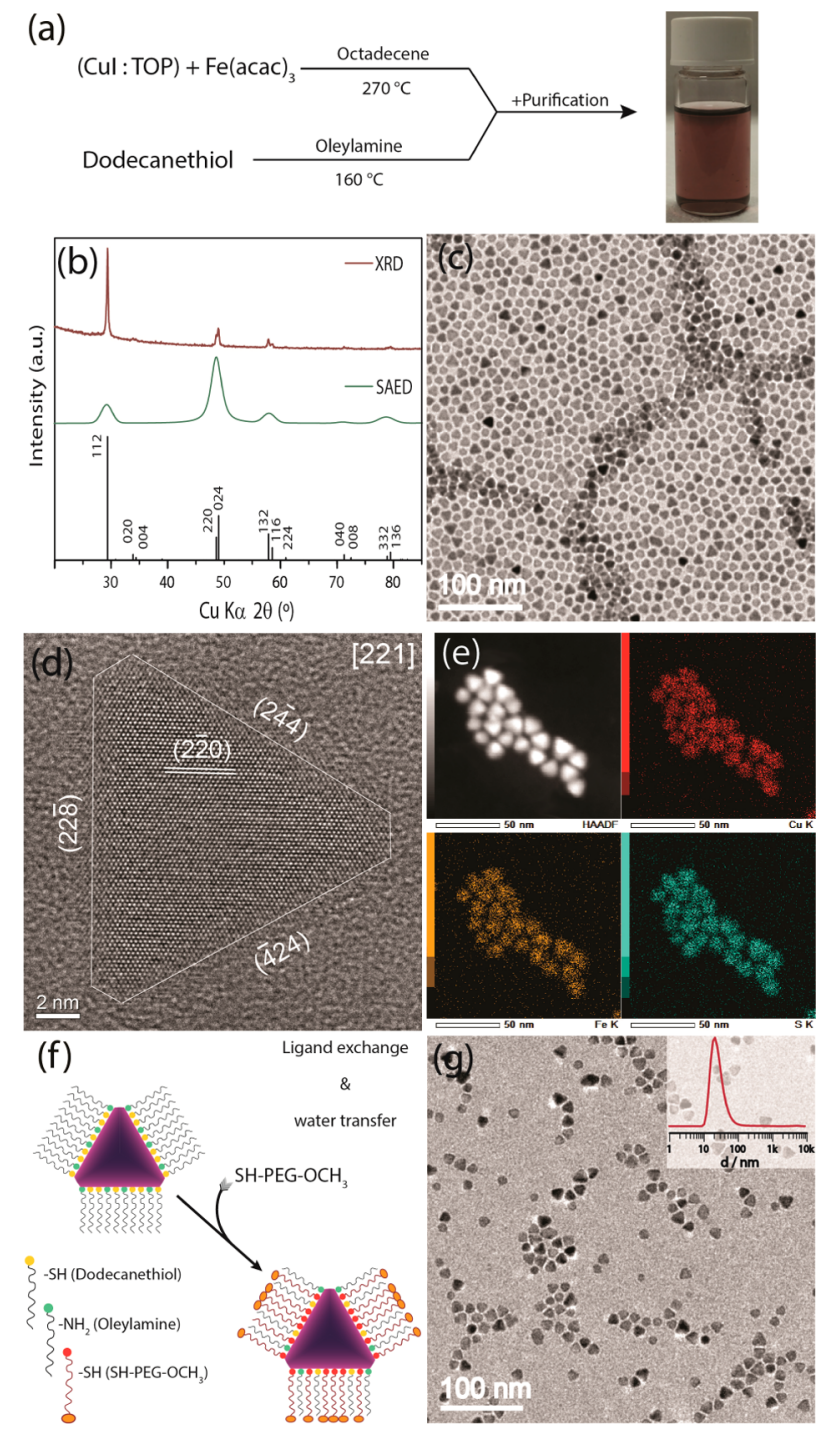

Figure 1. (a) Reaction scheme leading to the purple colored solution of $\mathrm{CuFeS}_{2} \mathrm{NCs}$ in chloroform (vial photograph). (b) XRD and azimuthally integrated SAED patterns for $\mathrm{CuFeS}_{2} \mathrm{NCs}$, in reference to

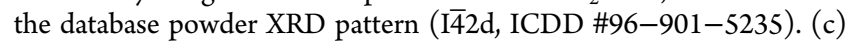
Overview BFTEM image of a set of NCs. (d) HRTEM image of a single NC viewed along the [221] direction. (e) HAADF-STEM image and corresponding STEM-EDS maps showing the distribution of the constituent elements. (f) Ligand exchange scheme for transferring the NCs to aqueous media using an amphiphilic polymer. (g) BFTEM image of $\mathrm{CuFeS}_{2} \mathrm{NCs}$ deposited from an aqueous solution with the hydrodynamic diameter (by volume), shown as an inset.

mixture) into the $\mathrm{Cu}-\mathrm{Fe}$ preinjection salt solution (degassed CuI-TOP-Fe(acac) ${ }_{3}$-ODE mixture), as confirmed by XRD patterns of the aliquots taken at different time intervals (Figure S1). Unlike Silvester et al., ${ }^{7}$ where a controlled conversion of amorphous iron(III) oxide particles into $\mathrm{CuFeS}_{2}$ was demonstrated, we have achieved a direct synthesis protocol. Monitoring the growth of the NCs revealed some interesting characteristics: the NCs exhibited a quasi-cuboctahedral shape within $1 \mathrm{~min}$ after the injection (average size $10.5 \pm 0.6 \mathrm{~nm}$; BFTEM images in Figure S1), which slowly ripened into the eventual pyramidal shape in the course of $15 \mathrm{~min}$ after injection, as shown in the representative overview BFTEM image in Figure 1, panel c. The latter stages (e.g., 25, 30, and 45 min; see Figure S1) were characterized by further ripening 
accompanied by truncation of the pyramid vertices leading to increased size dispersity. The unambiguous phase purity, however, could be ascertained only after a growth time of 15 min.

The elemental composition of the NCs was determined through ICP-OES analysis. The $\mathrm{Cu} / \mathrm{Fe}$ molar ratio of the NCs formed in the first few minutes $(1-5 \mathrm{~min})$ was slightly greater than 1 , that is, the NCs were Cu-rich. The desired value for $\mathrm{Cu} / \mathrm{Fe} / \mathrm{S}$ molar ratio of 1:1:2 was achieved after $15 \mathrm{~min}$ of growth, which lends further proof to phase purity. The NCs discussed henceforth are those that were allowed to grow for 15 $\mathrm{min}$ in the reaction mixture and were the most monodisperse ones, with an average edge length of $12.9( \pm 1.1) \mathrm{nm}$ (Figure 1c). An HRTEM image of a single NC viewed along the [221] crystal direction is shown in Figure 1, panel d, and the EDS elemental maps in Figure 1, panel e establish the distribution of the constituent elements in the set of NCs shown in the HAADF-STEM image in the upper left panel.

The hydrophobic $\mathrm{CuFeS}_{2} \mathrm{NCs}$, capped with a mixture of oleylamine/dodecanethiol, were transferred to the aqueous media by means of a ligand exchange procedure. The native ligands on the NC surface were exchanged with SH-PEG$\mathrm{OCH}_{3}$ molecules bearing a thiol $(-\mathrm{SH})$ moiety as anchoring group for the NC surface and a PEG chain with the methoxy $\left(-\mathrm{OCH}_{3}\right)$ headgroup as the hydrophilic unit. The PEG chains (attached to the NC surface and directed outward) enabled the dispersion of $\mathrm{CuFeS}_{2} \mathrm{NCs}$ in aqueous media, as schematically shown in Figure 1, panel f. The PEG-stabilized $\mathrm{CuFeS}_{2} \mathrm{NCs}$ $\left(\mathrm{CuFeS}_{2}-\mathrm{PEG}\right)$ retained their morphology and shape upon transferring to water, as is obvious from the BFTEM image shown in Figure 1, panel g. These NCs exhibited a narrow distribution of hydrodynamic diameters (DLS signal maximum at $21 \mathrm{~nm}$, inset in Figure 1g) with no other signals at bigger sizes, indicating the absence of aggregates. Thermogravimetric analysis (TGA) estimates an average of 95 PEG ligands per NC with the inorganic $\mathrm{CuFeS}_{2}$ core constituting $\sim 74 \%$ of the total mass of an individual NC (see Section S2 for details).

Optical Modeling/Characterization. The electronic structure of $\mathrm{CuFeS}_{2}$ was investigated using a DFT approach through a procedure similar to that presented in earlier works. $^{12,46}$ The calculated density of states (DOS) for the corresponding band structure of $\mathrm{CuFeS}_{2}$ (along the $\mathrm{Z}-\Gamma-\mathrm{X}$ line; Figure $\mathrm{S} 4 \mathrm{~b}$ ) is shown in Figure 2, panel a. An indirect band gap of $0.63 \mathrm{eV}$ separates the valence band from the lowest energy unoccupied states. These empty states form a narrow band, which is separated from a higher energy conduction band by a secondary energy gap of $1.20 \mathrm{eV}$. The overall band structure can hence be modeled in terms of a valence (VB), an intermediate (IB), and a conduction band (CB), similar to a previous theoretical work by Hamajima et al. ${ }^{13}$ The highest VB states are composed of mainly $\mathrm{Cu} d$ orbitals and $\mathrm{S} p$ orbitals, while the IB band consists of a large amount of states with minority spin, Fe d-orbitals character. The bottom of the $\mathrm{CB}$, on the other hand, has a clear s-character, with almost equal $S$, $\mathrm{Fe}$, and $\mathrm{Cu}$ contributions. The steady-state UV-Vis-NIR extinction spectrum of a dilute solution of $\mathrm{CuFeS}_{2} \mathrm{NCs}$ (Figure 2b) supports this description of the band structure as it features a broad continuous extinction starting from the NIR region $(\sim 1800 \mathrm{~nm})$ with an intense maximum at around $480 \mathrm{~nm}$ accompanied by a broad shoulder centered at around $950 \mathrm{~nm}$. These two distinct absorption bands, as well as the nonzero absorption in the whole NIR region, are attributed to the electronic transitions from the VB to the IB. (a)

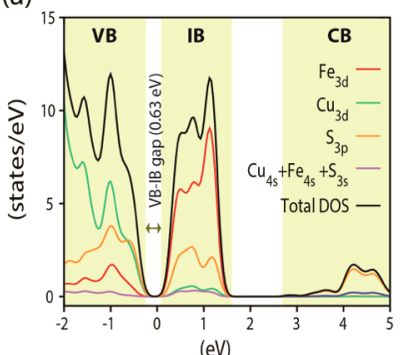

(b)

(c)
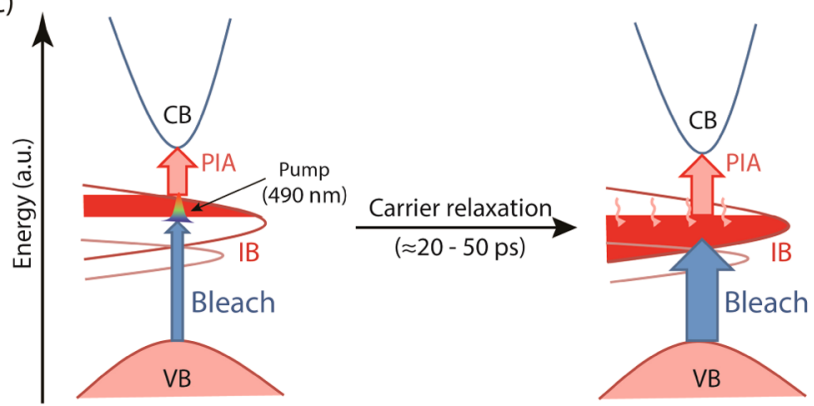

(d)

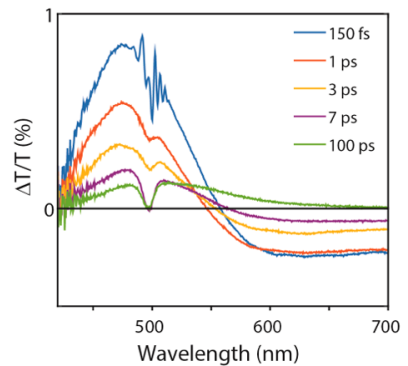

(e)

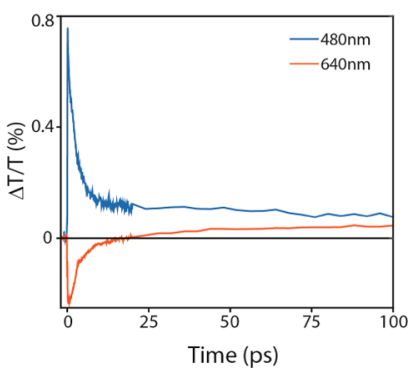

Figure 2. (a) Calculated DOS for the corresponding electronic band structure. (b) Steady state absorption spectrum of $\mathrm{CuFeS}_{2} \mathrm{NCs}$ (shaded area depicting the region of transient absorption measurements). (c) Tentative sketches showing the optical excitations and relaxation processes in $\mathrm{CuFeS}_{2}$. (d) Transient absorption measurements in the range of $0-100$ ps. The spectrum is contributed by a strong bleach signature $(430-550 \mathrm{~nm})$ and a photoinduced absorption (PIA) signal $(550-750 \mathrm{~nm})$ at longer wavelengths. A strong broadening of the bleach signal over time is observed. (e) Dynamics at 480 and $640 \mathrm{~nm}$, depicted in blue and orange, respectively.

We carried out TA measurements to elucidate the processes of carrier excitation and relaxation in these NCs. We probed the $430-700 \mathrm{~nm}$ spectral range (Figure $2 \mathrm{~b}$, shaded region) by pumping at $490 \mathrm{~nm}$ into the strong absorption band of $\mathrm{CuFeS}_{2}$, which corresponds to transitions from the VB to the higher IB (see sketches in Figure 2c). We decided to pump just off the maximum of the transition at $480 \mathrm{~nm}$ to minimize the perturbation of the signal by pump scattering. Because of measurement constraints, we focused only on the higher energy VB-IB transition at $480 \mathrm{~nm}$. However, we are confident that our findings, which are detailed below, should equally apply when investigating the transition from the VB to the lower IB (at $950 \mathrm{~nm}$ ) possible by an IR pulse. The main findings from these experiments are that the carriers undergo fast relaxation within the IB observed on a time scale of approximately 20-50 ps, before interband relaxation occurs. This fast initial relaxation is ascribed to carrier cooling within the broad IB via the interaction with the lattice and the emission of phonons. This results in the efficient heating of the $\mathrm{NC}$ as observed and 
exploited by us for PT (see further). Details of the TA measurements are discussed in the following paragraphs.

Pumping at $490 \mathrm{~nm}$ into the strong optical absorption band of $\mathrm{CuFeS}_{2}$ results in a bleach around $430-550 \mathrm{~nm}$ coinciding with the region of the highest absorption in the steady state spectra and a broad photo induced absorption signal (PIA) further red. The corresponding TA spectra in the range of $0-$ 100 ps are given in Figure 2, panel d. The decay dynamics at the bleach maximum ( $480 \mathrm{~nm}$, blue curve in Figure 2e) are governed by a two-step decay with a fast component of around 20-50 ps and a slower component of several hundreds of picoseconds. The PIA signal at $640 \mathrm{~nm}$ (red curve in Figure 2e) shows a similar decay dynamics and an initial fast rise of less than 1 ps. After about 20-50 ps, the negative PIA signal turns to a positive signal. Normalization of the spectra (see Figure S5 in the Supporting Information) shows that the spectral shape of the bleach and the PIA is retained during the first 6 ps. Thereafter, the spectrum broadens and shifts to the red. After about $20-50$ ps, the transient spectrum is composed of solely a bleach signal covering nearly the entire region of investigation, which shows a shoulder to the red of the bleach signal.

The excitation and relaxation processes occurring within the first $20-50$ ps are depicted schematically in the sketches of Figure 2, panel c, on the band structure of $\mathrm{CuFeS}_{2}$ composed of the VB, a broad, initially empty IB, and the CB. The results have been interpreted in the following way: the pump pulse at $490 \mathrm{~nm}$ (rainbow colored pulse in the left sketch) leads to the occupation of states in the IB (red shaded area in Figure 2c), which is narrow in comparison to the width of the IB. An immediate bleach is induced (blue arrow), as these states are occupied and not available for further VB to IB transitions. Simultaneously, a PIA signal is evolving nearly immediately between the IB and the CB (red arrow) due to the temporary occupation of states in the IB. The rather broad PIA signal reflects the manifold of carriers and states involved in this transition within the IB and the $\mathrm{CB}$. The short rise time is a result of the time it takes to fill states in the IB and is within the time resolution of our system. Within the first $20-50 \mathrm{ps}$, the carriers thermalize to the edge of the IB (indicated as small wavy arrows in right sketch of Figure 2c), which results in a red shift and broadening of the bleach signal (blue arrow). The shoulder to the red of the bleach uncovers the still present negative PIA signal further red due to IB-CB transitions (red arrow). After cooling of the excited carriers within the first 20$50 \mathrm{ps}$, a slower interband relaxation is observed (slower component of several hundreds of picoseconds, Figure 2e). Our results confirm that within the first $20-50$ ps carrier cooling occurs by transferring the excess energy of the excited carriers in the IB to the lattice of the NC resulting in the heating of the $\mathrm{NC}$, before, on a slower time scale of several hundreds of picoseconds, the interband relaxation takes place. Our results highlight the importance of the unique band structure of $\mathrm{CuFeS}_{2}$ constituting a broad IB for efficient light-to-heat conversion.

Photothermal Conversion and Stability Studies. The extinction spectrum of an aqueous solution of PEG-coated NCs $\left(\mathrm{CuFeS}_{2}-\mathrm{PEG} \mathrm{NCs}\right.$; Figure S6) had features that closely matched those of the as-synthesized NCs in chloroform (Figure $2 \mathrm{~b})$. The continuous absorption in the NIR region, particularly within the first biological window $(650-900 \mathrm{~nm})$, accompanied by a high molar attenuation coefficient $\left(\varepsilon=5.2 \times 10^{6} \mathrm{M}^{-1} \mathrm{~cm}^{-1}\right.$ at $808 \mathrm{~nm}$; section S5 in Supporting Information), makes them potentially suitable candidates for photothermal applications.
The molar attenuation coefficient for the $\mathrm{CuFeS}_{2}-\mathrm{NC}$ here measured was comparable to that of other chalcogenide NCs, such as $\mathrm{CuS}$ nanoplatelets, ${ }^{47} \mathrm{Cu}_{2-x} \mathrm{Se} \mathrm{NCs},{ }^{24}$ and $\mathrm{Cu}_{9} \mathrm{~S}_{5} \mathrm{NCs},{ }^{48}$ and it is at least one order of magnitude higher than that of standard photosensitizer dyes used as potential photothermal agents. ${ }^{49}$ The molar attenuation coefficient can also be expressed as the per particle absorption cross-section using $C_{\mathrm{abs}}=2303 \varepsilon / N_{\mathrm{A}}\left(N_{\mathrm{A}}\right.$ is the Avogadro constant $)$ considering the fact that scattering contribution to extinction for small NCs can generally be neglected due to their small size with respect to the probing wavelength ( $808 \mathrm{~nm}$ in this case).$^{50}$ The per particle absorption cross-section for $\mathrm{CuFeS}_{2}-\mathrm{PEG}$ NCs at 808 $\mathrm{nm}$ is $2 \times 10^{-14} \mathrm{~cm}^{2}$.

A remarkable temperature increase was recorded upon irradiation of an aqueous solution of $\mathrm{CuFeS}_{2}-\mathrm{PEG}$ NCs with an $808 \mathrm{~nm}$ laser. The extent of light to heat conversion by a sensitizer is typically expressed as the so-called PTE $\eta$, defined as the fraction of absorbed light that has been converted to heat and is expressed as follows (as per Roper et $\mathrm{al}^{43}$ ):

$$
\eta=\frac{h S\left(T_{\max }-T_{\mathrm{amb}}\right)-Q_{\mathrm{Dis}}}{I\left(1-10^{-A 808}\right)}
$$

where $h$ is the heat transfer coefficient, $S$ is the surface area of the container in which the NC solution was placed, and $T_{\max }$ and $T_{\text {amb }}$ represent the maximum equilibrium temperature reached by the solution and the ambient temperature, respectively. $Q_{D i s}$ represents the heat dissipated by the solvent and the quartz cuvette itself upon light absorption, $I$ is the incident laser power, and $A 808$ is the extinction of the sample at $808 \mathrm{~nm}$. As evident from the equation, the PTE represents the amount of heat generated per incident light power and was estimated to be $49 \%$ in our case (see Figure S 8 b and section S6 of the Supporting Information for details on calculations). This value is in line with previous reports on chalcogenide NCs. Overall, we assign this high efficiency to the large molar attenuation coefficient of these NCs and to the thermalization of carriers that are excited from the VB to the IB. The presence of numerous direct $\mathrm{VB}-\mathrm{IB}$ transitions at this energy difference $(808 \mathrm{~nm}, 1.53 \mathrm{eV})$, as evident from the electronic band structure shown in Figure S4b, contributes to the high molar attenuation coefficient. Additionally, the absence of photoluminescence in our NCs, both in those as-synthesized and after water transfer, and which can be due to various reasons (presence of trap or defect states, indirect gap between the top of the VB and the bottom of the IB), indicates that even this intergap relaxation must occur primarily by thermal processes.

It was important to evaluate the stability of $\mathrm{CuFeS}_{2}-\mathrm{PEG}$ NCs in physiological conditions, which in turn can affect the photothermal performance of the NCs under the laser. Accordingly, a stability test was performed wherein NC solutions with total $\mathrm{Cu}$ concentration (henceforth indicated as $[\mathrm{Cu}])$ of $10 \mathrm{ppm}$ were incubated in a complete cell culture medium (DMEM) at $37^{\circ} \mathrm{C}$ for $24 \mathrm{~h}$, and the time evolution of hydrodynamic diameter was monitored through DLS.

$\mathrm{CuFeS}_{2}-\mathrm{PEG} \mathrm{NCs}$, either in water or in complete DMEM, were stable at $37^{\circ} \mathrm{C}$ even after $24 \mathrm{~h}$, with no sign of aggregation or precipitation (the spectral characteristics and color of the NC solution did not change and no aggregation was observed at the bottom of the vials, see Figure S10). A similar test was also performed under laser illumination to ascertain the colloidal stability of $\mathrm{CuFeS}_{2}-\mathrm{PEG}$ NCs during heating cycles. A NC solution at $15 \mathrm{ppm}[\mathrm{Cu}]$ was placed inside a DLS cuvette 
(a)

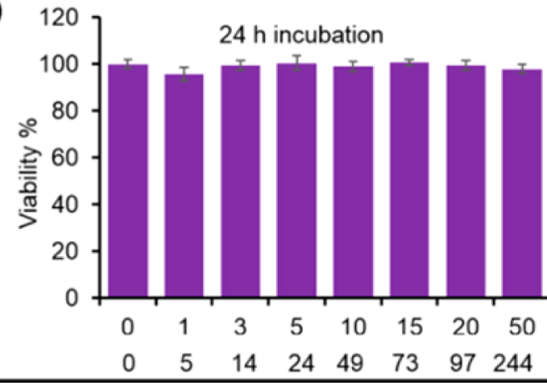

(b)

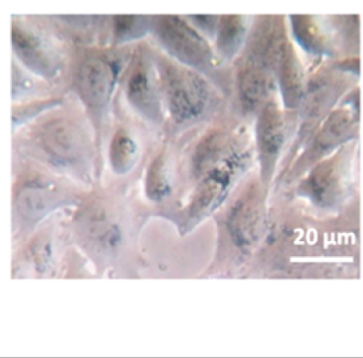

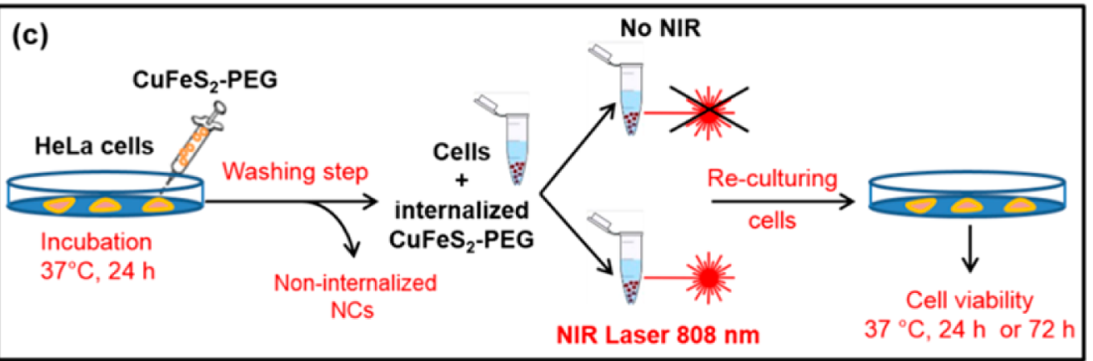

(d)

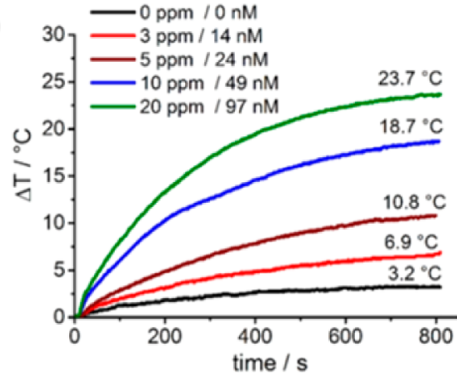

(f)

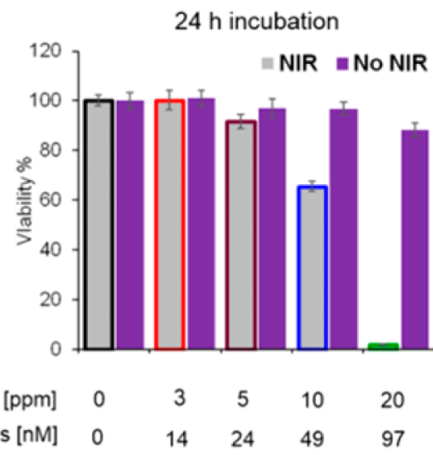

(e)

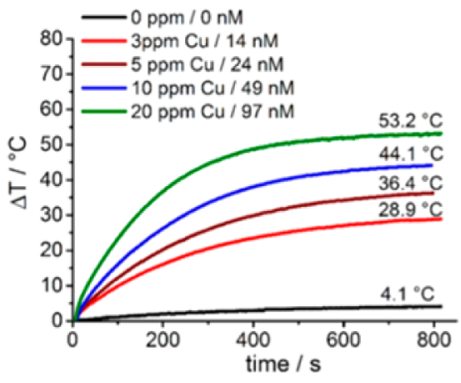

(g)

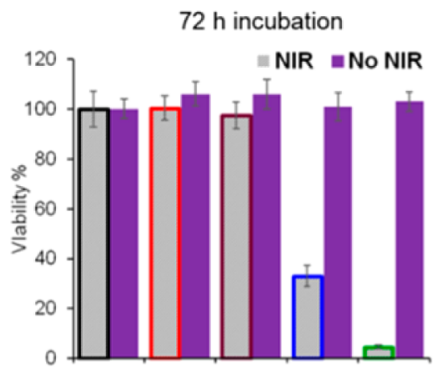

$\begin{array}{cccccc}\text { Cu [ppm] } & 0 & 3 & 5 & 10 & 20 \\ \text { NCs [nM] } & 0 & 14 & 24 & 49 & 97\end{array}$

Figure 3. (a) Cell viability test after $24 \mathrm{~h}$ of incubation of $\mathrm{HeLa}$ cells with solutions of $\mathrm{CuFeS}_{2}-\mathrm{PEG}$ NCs under radiationless conditions and at different NC concentrations. (b) Optical image of HeLa cells after exposure to $\mathrm{NCs}$ for $24 \mathrm{~h}$ at $37{ }^{\circ} \mathrm{C}, 5 \% \mathrm{CO}_{2}$ at $[\mathrm{Cu}]$ of 20 ppm: the bluish spots are internalized NCs. (c) Schematic summary of the cell in vitro photothermal experiment. (d) Temperature profile under laser irradiation of HeLa cells in medium solution previously incubated at varying amounts of initial administered NCs for $24 \mathrm{~h}$ at $37^{\circ} \mathrm{C}$. (e) Temperature profile under laser irradiation of just NCs dissolved in cell medium at different concentrations and corresponding to the initial NC doses administered to the cells. The initial temperature for both panels $\mathrm{d}$ and e was around $30^{\circ} \mathrm{C}$. Experiment of laser irradiation effects on cells (f and g). HeLa cells were exposed for $24 \mathrm{~h}$ to $\mathrm{CuFeS}_{2}-\mathrm{NCs}$; after the cells were washed from the nonassociated $\mathrm{CuFeS}_{2}-\mathrm{NCs}$, the cells collected in $0.5 \mathrm{~mL}$ medium were irradiated by the laser for $13 \mathrm{~min}$ (NIR) or they were kept at RT for the same time interval (No NIR). On the recultured cells, viability was assessed after (f) $24 \mathrm{~h}$ and (g) $72 \mathrm{~h}$. By the comparison of cells exposed and not exposed to the NIR, it is clear that the irradiation-induced cytotoxicity increases in a dosedependent manner. The cell viability was measured using control cells (i.e., cells not incubated with NCs) as a reference.

and irradiated with the $808 \mathrm{~nm}$ laser for several cycles. DLS recorded after each irradiation cycle showed no significant changes, which confirmed the stability of these NCs under laser irradiation (see Figure S11 and Section S8 for more details). The heating-cooling temperature profiles under laser irradiation were consistently reproduced over five consecutive cycles, which showed the sample stability under repetitive heating cycles (Figure S12).

Cell Studies. An ideal photothermal agent should exhibit minimal intrinsic toxicity to the living cells under radiationless conditions. To clear this point in the present case, the biocompatibility of the $\mathrm{CuFeS}_{2}-\mathrm{PEG}$ NCs was evaluated on HeLa cells by means of a PB assay. Solutions at various concentrations of $\mathrm{CuFeS}_{2}-\mathrm{PEG}$ NCs, in a range from 5-244 $\mathrm{nM}$ (corresponding to $[\mathrm{Cu}]$ ranging from 1 to $50 \mathrm{ppm}$ ), were administered to HeLa cells, and the toxicity was evaluated after 24 and $72 \mathrm{~h}$ of incubation at $37^{\circ} \mathrm{C}$. The cell viability after $24 \mathrm{~h}$ was not affected by the presence of the NCs, as no sign of cytotoxicity was detected in the whole concentration range analyzed (viability values above $97 \%$ were recorded, Figure 3a), 
in line with results reported for other chalcogenide NCs. ${ }^{51}$ The internalized NCs were discernible under the optical microscope at $[\mathrm{Cu}]$ feeds above $10 \mathrm{ppm}$ (bluish spots in Figure $3 \mathrm{~b}$ ). However, at $72 \mathrm{~h}$ of continuous NC exposure, the viability decreased with increasing concentration of the NC: the viability was above $80 \%$ for [Cu] up to $5 \mathrm{ppm}$, while significant toxicity was observed when the cells were continuously exposed to NC solutions with $[\mathrm{Cu}]$ higher than $10 \mathrm{ppm}$ (Figure S13). A similar toxicity trend was also confirmed when the same NCs were tested on another tumor cell line, as for instance the IGROV 1 cells (Figure S14). The low toxicity of the $\mathrm{CuFeS}_{2}-\mathrm{PEG}$ NCs in the range from 3-10 ppm motivated us to study their photothermal activity as well (as schematically shown in Figure $3 c)$. In an in vitro test, cultured cells were first exposed to NC solutions at different $[\mathrm{Cu}]$ doses $(3,5,10$, and $20 \mathrm{ppm})$ for 24 $\mathrm{h}$ at $37^{\circ} \mathrm{C}$ to enable $\mathrm{NC}$ internalization by the cells before the laser treatment was performed. After the non-internalized NCs were rinsed off, one fraction of the cells was detached and resuspended in a fresh medium for exposure to NIR radiation $\left(1.14 \mathrm{~W}, 3.1 \mathrm{~W} \mathrm{~cm}^{-2}\right.$, spot size $0.36 \mathrm{~cm}^{2}, 13$ min irradiation time). The other fraction, used as control, was kept for the same time span as the irradiated fraction at room temperature (around $30^{\circ} \mathrm{C}$ ) but was not irradiated (this sample is indicated as "No NIR" in Figure 3c). Upon laser irradiation, a rise in the temperature of the whole cell sample solution was observed, even for initial $[\mathrm{Cu}]$ values as low as $5 \mathrm{ppm}$. For cells exposed to $[\mathrm{Cu}]$ doses above $5 \mathrm{ppm}$, the temperature increase was significantly higher than that of pure water irradiated with the same laser at equivalent power and time.

The temperature rise was much more pronounced at increasing amounts of NC exposure, which suggested a progressively higher NC internalization by the cells at higher exposure dose, as seen from Figure 3, panel d. The maximum temperature reached on the cell samples was always lower than that of the solutions prepared by dissolving an equal amount of NCs in $0.5 \mathrm{~mL}$ of DMEM (Figure 3e). This indicates that the amount of NCs internalized by the cells is definitely lower than that of the initially administrated dose, and indeed only a small fraction is taken up by the cells. After laser irradiation, the cells were recultured, and the viability was assessed after 24 and $72 \mathrm{~h}$ of the laser treatment. For initial $[\mathrm{Cu}]$ doses of $10 \mathrm{ppm}$ or above, a surge in cytotoxicity for Hela cells could already be distinguished after $24 \mathrm{~h}$ on the irradiated samples when compared to the non-irradiated control sample, as shown in Figure 3, panel f. After $72 \mathrm{~h}$, the rise in cytotoxicity was much more pronounced at all the NC doses tested, which indicated a permanent cell damage rather than a bare acute toxic effect (Figure 3g). The control cells, containing internalized NCs but not irradiated, did not show signs of toxicity ("No NIR" in Figure $3 \mathrm{~g}$ ). Another set of control cells, irradiated with the laser but not incubated with the NCs, exhibited $100 \%$ viability. This stands as a proof that the laser light in the conditions of the experiments used $\left(1.14 \mathrm{~W}, 3.1 \mathrm{~W} \mathrm{~cm}^{-2}\right.$ spot size $0.36 \mathrm{~cm}^{2}, 13$ min irradiation time) did not affect the cell viability unless it was partially absorbed by the NCs and was then converted into heat.

In an in vivo photothermal treatment of a tumor, often the photothermal agents are intratumorally injected before the tumor area is exposed to the laser treatment. This approach minimizes the amount of photosensitizer needed for an effective photothermal therapy, as it is delivered locally and not through the bloodstream. A laser irradiation experiment, hence, was designed to mimic the case of an intratumoral injection of NCs by choosing the minimal dose of $\mathrm{CuFeS}_{2}-$ PEG NCs that is required to increase the temperature above 46 ${ }^{\circ} \mathrm{C}$ (which is considered high enough to induce tumor cell damage) and that at the same time does not manifest any sign of toxicity in dark conditions (laser power of $3.1 \mathrm{~W} \mathrm{~cm}$, Figure S15). The temperature profiles (Figure 3e) and toxicity tests over prolonged exposure times (Figures S13 and S14) indicate that this dose corresponds to $[\mathrm{Cu}]$ of $3 \mathrm{ppm}$. A cell viability above $97 \%$ was recorded at this dose, even after $72 \mathrm{~h}$ of continuous NC exposure. Upon irradiating a NC solution at this dose, the temperature rose from the initial 30 to $58.9{ }^{\circ} \mathrm{C}$ (corresponding to a $\Delta T$ of $28.9{ }^{\circ} \mathrm{C}$ ), as shown in Figure 3, panel e.

After having identified $3 \mathrm{ppm}$ in $[\mathrm{Cu}]$ as the optimal dose, we performed an in vitro experiment in which the cells were exposed to this dose of NCs for $24 \mathrm{~h}$. In one case, the cells were not washed from the non-internalized NCs prior to irradiation with the laser. In another case, the cells underwent the same incubation conditions with the NCs, but they were rinsed before being irradiated. A control sample was represented by cells that were not incubated with NCs but were irradiated with the laser. Obviously, irradiation of the unwashed sample caused a much higher increase in temperature, from 28 to $50{ }^{\circ} \mathrm{C}$ after $13 \mathrm{~min}$ of exposure (Figure 4a, blue line), than in the washed sample (Figure 4a, red line), which behaved in practice as the control sample (Figure 4a, black line). The cells, after the different treatments, were recultured, and their viability was assessed after $72 \mathrm{~h}$. The viability of the "unwashed" sample was less than $5 \%$ (Figure $4 \mathrm{~b}$, histogram marked with a blue line), while that of the "washed" sample was close to $100 \%$ (Figure 4, panel $b$, histogram marked with a red line), comparable to that of the control sample (Figure 4, panel b, histogram marked with a black line). For each of these three samples, we also ran a parallel experiment consisting in treating cells in the same manner but not subjecting them to laser irradiation. The viability for these parallel samples is reported in purple histograms in Figure 4, panel b (No-NIR). In these latter samples, no toxicity effects were detectable. The overall conclusion from these experiments is that $3 \mathrm{ppm}[\mathrm{Cu}]$ (corresponding to $14 \mathrm{nM}$ in NCs) represents a concentration that does not cause any toxicity effects on the cells, even after prolonged exposure, but it does become toxic under irradiation.

We performed an additional qualitative experiment to test cell viability after NIR irradiation based on the simultaneous determination of live and dead cells through fluorescence imaging using calcein AM (green) and EthD-1 (red) as dyes, respectively, as presented in Figure 4, panels $\mathrm{c}$ and d. Briefly, cells exposed to $3 \mathrm{ppm}$ in $[\mathrm{Cu}]$ concentration of $\mathrm{NC}$ were first irradiated and then recultured to analyze their viability (after 5 h) under a fluorescence confocal microscope in comparison to cells irradiated without the exposure to the NCs. As expected, irradiated cells treated without the NCs were healthy and attached to the substrate (green color, Figure 4c), whereas most of the cells treated with $3 \mathrm{ppm}$ in $[\mathrm{Cu}]$ dose, upon irradiation were dead (red color, Figure $4 \mathrm{~d}$ ) or were suffering (orange, Figure $4 \mathrm{~d}$ ). Furthermore, a lower number of irradiated cells exposed to the NCs was able to adhere to the multiwall (fewer fluorescent spots in Figure 4d than in Figure 4c).

\section{CONCLUSIONS}

We have described a colloidal synthesis of monodisperse and phase-pure chalcopyrite $\left(\mathrm{CuFeS}_{2}\right) \mathrm{NCs}$, which are characterized by an absorption spanning almost the entire visible and 

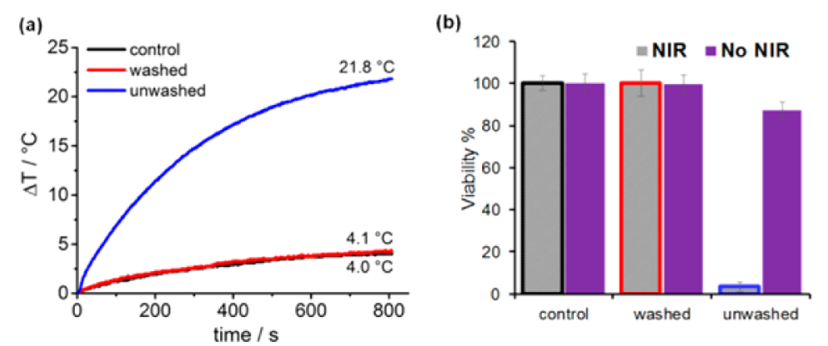

(c)
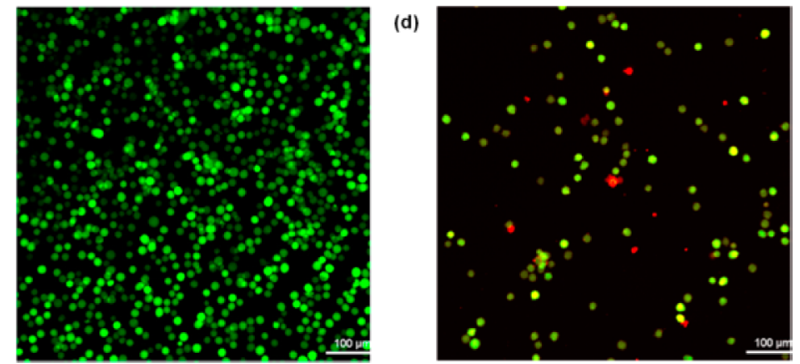

Figure 4. Identification of the minimal dose of $\mathrm{CuFeS}_{2} \mathrm{NCs}$ needed to produce cell damage only under laser irradiation. (a) Temperature profile under irradiation of cell samples incubated with $\mathrm{CuFeS}_{2}-\mathrm{PEG}$ NCs (at a dose of $3 \mathrm{ppm}[\mathrm{Cu}]$ for $24 \mathrm{~h}$ ) under irradiation. The red curve refers to the fraction of cells "washed" from noninternalized NCs prior to irradiation, whereas the blue curve refers to the cells that were "unwashed" from the NCs. The black line corresponds to the effect of irradiation of the control sample (cells not incubated with the NCs). In the unwashed sample, the noninternalized NCs contribute significantly to raise the cell temperature, which reached $50{ }^{\circ} \mathrm{C}$ (the initial solution temperature was around $28{ }^{\circ} \mathrm{C}$ ). (b) Cell viability recorded on the cells after laser treatment and after reculturing for 72 $\mathrm{h}$, which indicated the high toxicity of the NCs in the case of "unwashed" sample. For each of the three cases, the purple histogram reports, as a control, the viability of cells treated in the same manner but not subjected to laser irradiation. Fluorescence confocal images of the irradiated cells (c) without and (d) with NCs (at 3 ppm of [Cu]) after staining with calcein $\mathrm{AM}$ (green) and $\mathrm{EthD}-1$ (red). The green color signifies healthy cells (as in panel c, without NCs), which decrease in number on irradiation in the presence of NCs with an accompanying rise in the number of damaged/dead cells signified by the presence of orange/red cells, as shown in panel $\mathrm{d}$.

NIR regions, with broad maxima at 480 and $950 \mathrm{~nm}$. The presence of an IB in the electronic band structure (as supported by DFT and TA spectroscopy) with a relatively small indirect VB-IB gap makes these NCs suitable for light-to-heat conversion in the biological window of 650-900 nm. Consequently, chalcopyrite NCs exhibit enhanced PT efficiency of $49 \%$ under laser radiation of $808 \mathrm{~nm}$. In addition, the small hydrodynamic diameter of these NCs coupled with high natural abundance of the chalcopyrite mineral (and hence of the metals $\mathrm{Cu}$ and $\mathrm{Fe}$ ) makes them excellent candidates for photothermal therapy. Tumor cell annihilation upon laser irradiation in the presence of $\mathrm{CuFeS}_{2} \mathrm{NCs}$ demonstrates their therapeutic ability. The NCs are stable in biological liquids so that, in principle, they can be injected into organisms, either intratumorally or intravenously, which will constitute our future work.

It is evident from our studies that the otherwise detrimental effects of iron on photovoltaic applications can be a boon for applications like photothermal therapy. The same concept of introducing deep Fe intermediate levels in conventional semiconductors can be used to design a new class of photothermal agents, which operate through a different light to heat conversion mechanism than that for plasmonic NCs like gold and copper chalcogenides. Indeed, iron doping in other chalcopyrite type semiconductors (like $\mathrm{CuGaS}_{2}$ and $\mathrm{CuAlS}{ }_{2}$ ) introduces similar effects as in $\mathrm{CuFeS}_{2}$.

\section{ASSOCIATED CONTENT}

\section{S Supporting Information}

The Supporting Information is available free of charge on the ACS Publications website at DOI: 10.1021/acs.chemmater.6b02192.

Additional experiments on NC growth monitoring; TGA of $\mathrm{CuFeS}_{2}-\mathrm{PEG} \mathrm{NCs}$; theoretical electronic band structure calculations; normalized TA spectra of the NCs; calculations of molar attenuation coefficient $(\varepsilon)$ and PT efficiency; DLS data on NC stability in physiological conditions; colloidal stability of NCs under laser irradiation; reversibility of the heatingprofile curves; additional data on cell viability and photothermal experiments (PDF)

\section{AUTHOR INFORMATION}

\section{Corresponding Authors}

*E-mail: sandeep.ghosh@iit.it.

*E-mail: teresa.pellegrino@iit.it.

*E-mail: liberato.manna@iit.it.

\section{Author Contributions}

$\square$ These authors contributed equally.

Notes

The authors declare no competing financial interest.

\section{ACKNOWLEDGMENTS}

The research leading to these results has received funding from the FP7 under Grant Agreement No. 614897 (ERC Consolidator Grant "TRANS-NANO") and from the Horizon 2020 under Grant Agreement No. 678109 (ERC starting grant "ICARO"). The IIT platform CompuNet and the Italian Supercomputer Center CINECA (Bologna, Italy) are acknowledged for the computational resources.

\section{REFERENCES}

(1) Teranishi, T. Magnetic and Electric Properties of Chalcopyrite. J. Phys. Soc. Jpn. 1961, 16, 1881-1887.

(2) Sherbini, M. A. E.; Yousef, Y. L. The Effect of Cooling and of Magnetic Fields on Crystal Rectification. Proc. Phys. Soc. 1939, 51, 449-455.

(3) Austin, I. G.; Goodman, C. H. L.; Pengelly, A. E. New Semiconductors with the Chalcopyrite Structure. J. Electrochem. Soc. 1956, 103, 609-610.

(4) Knight, K. S.; Marshall, W. G.; Zochowski, S. W. The LowTemperature and High-Pressure Thermoelastic and Structural Properties of Chalcopyrite, $\mathrm{CuFeS}_{2}$. Can. Mineral. 2011, 49, 1015-1034.

(5) Aldakov, D.; Lefrancois, A.; Reiss, P. Ternary and Quaternary Metal Chalcogenide Nanocrystals: Synthesis, Properties and Applications. J. Mater. Chem. C 2013, 1, 3756-3776.

(6) Gabka, G.; Bujak, P.; Zukrowski, J.; Zabost, D.; Kotwica, K.; Malinowska, K.; Ostrowski, A.; Wielgus, I.; Lisowski, W.; Sobczak, J. W.; Przybylski, M.; Pron, A. Non-Injection Synthesis of Monodisperse Cu-Fe-S Nanocrystals and Their Size Dependent Properties. Phys. Chem. Chem. Phys. 2016, 18, 15091-15101.

(7) Silvester, E. J.; Healy, T. W.; Grieser, F.; Sexton, B. A. Hydrothermal Preparation and Characterization of Optically Transparent Colloidal Chalcopyrite $\left(\mathrm{CuFeS}_{2}\right)$. Langmuir 1991, 7, 19-22.

(8) Oguchi, T.; Sato, K.; Teranishi, T. Optical Reflectivity Spectrum of a $\mathrm{CuFeS}_{2}$ Single Crystal. J. Phys. Soc. Jpn. 1980, 48, 123-128. 
(9) Wang, Y.-H. A.; Bao, N.; Gupta, A. Shape-Controlled Synthesis of Semiconducting $\mathrm{CuFeS}_{2}$ Nanocrystals. Solid State Sci. 2010, 12, 387390.

(10) Liang, D.; Ma, R.; Jiao, S.; Pang, G.; Feng, S. A Facile Synthetic Approach for Copper Iron Sulfide Nanocrystals with Enhanced Thermoelectric Performance. Nanoscale 2012, 4, 6265-6268.

(11) Xie, Y.; Riedinger, A.; Prato, M.; Casu, A.; Genovese, A.; Guardia, P.; Sottini, S.; Sangregorio, C.; Miszta, K.; Ghosh, S.; Pellegrino, T.; Manna, L. Copper Sulfide Nanocrystals with Tunable Composition by Reduction of Covellite Nanocrystals with $\mathrm{Cu}^{+}$Ions. J. Am. Chem. Soc. 2013, 135, 17630-17637.

(12) Fukushima, T.; Katayama-Yoshida, H.; Uede, H.; Takawashi, Y.; Nakanishi, A.; Sato, K. Computational Materials Design of Negative Effective U System in Hole-Doped Chalcopyrite $\mathrm{CuFeS}_{2}$. J. Phys.: Condens. Matter 2014, 26, 355502-355510.

(13) Hamajima, T.; Kambara, T.; Gondaira, K. I.; Oguchi, T. SelfConsistent Electronic Structures of Magnetic Semiconductors by a Discrete Variational X $\alpha$ Calculation. III. Chalcopyrite $\mathrm{CuFeS}_{2}$. Phys. Rev. B: Condens. Matter Mater. Phys. 1981, 24, 3349-3353.

(14) Tossell, J. A.; Urch, D. S.; Vaughan, D. J.; Wiech, G. The Electronic Structure of $\mathrm{CuFeS}_{2}$, Chalcopyrite, from X-Ray Emission and X-Ray Photoelectron Spectroscopy and $\mathrm{X} \alpha$ Calculations. J. Chem. Phys. 1982, 77, 77-82.

(15) Kvande, R.; Geerligs, L. J.; Coletti, G.; Arnberg, L.; Di Sabatino, M.; Øvrelid, E. J.; Swanson, C. C. Distribution of Iron in Multicrystalline Silicon Ingots. J. Appl. Phys. 2008, 104, 064905.

(16) Teranishi, T.; Sato, K.; Kondo, K. i. Optical Properties of a Magnetic Semiconductor: Chalcopyrite $\mathrm{CuFeS}_{2}$.: I. Absorption Spectra of $\mathrm{CuFeS}_{2}$ and Fe-Doped $\mathrm{CuAlS}_{2}$ and $\mathrm{CuGaS}_{2}$. J. Phys. Soc. Jpn. 1974, 36, 1618-1624.

(17) Cheng, L.; Wang, C.; Feng, L.; Yang, K.; Liu, Z. Functional Nanomaterials for Phototherapies of Cancer. Chem. Rev. 2014, 114, 10869-10939.

(18) Jaque, D.; Martinez Maestro, L.; del Rosal, B.; Haro-Gonzalez, P.; Benayas, A.; Plaza, J. L.; Martin Rodriguez, E.; Garcia Sole, J. Nanoparticles for Photothermal Therapies. Nanoscale 2014, 6, 94949530.

(19) Weissleder, R. A Clearer Vision for in Vivo Imaging. Nat. Biotechnol. 2001, 19, 316-317.

(20) Huang, X.; El-Sayed, I. H.; Qian, W.; El-Sayed, M. A. Cancer Cell Imaging and Photothermal Therapy in the Near-Infrared Region by Using Gold Nanorods. J. Am. Chem. Soc. 2006, 128, 2115-2120.

(21) Chen, J.; Yang, M.; Zhang, Q.; Cho, E. C.; Cobley, C. M.; Kim, C.; Glaus, C.; Wang, L. V.; Welch, M. J.; Xia, Y. Gold Nanocages: A Novel Class of Multifunctional Nanomaterials for Theranostic Applications. Adv. Funct. Mater. 2010, 20, 3684-3694.

(22) Shi, P.; Liu, Z.; Dong, K.; Ju, E.; Ren, J.; Du, Y.; Li, Z.; Qu, X. A Smart "Sense-Act-Treat" System: Combining a Ratiometric Ph Sensor with a Near Infrared Therapeutic Gold Nanocage. Adv. Mater. 2014, 26, 6635-6641.

(23) Liu, H.; Chen, D.; Li, L.; Liu, T.; Tan, L.; Wu, X.; Tang, F. Multifunctional Gold Nanoshells on Silica Nanorattles: A Platform for the Combination of Photothermal Therapy and Chemotherapy with Low Systemic Toxicity. Angew. Chem., Int. Ed. 2011, 50, 891-895.

(24) Hessel, C. M.; Pattani, V. P.; Rasch, M.; Panthani, M. G.; Koo, B.; Tunnell, J. W.; Korgel, B. A. Copper Selenide Nanocrystals for Photothermal Therapy. Nano Lett. 2011, 11, 2560-2566.

(25) Li, W. H.; Zamani, R.; Rivera Gil, P.; Pelaz, B.; Ibanez, M.; Cadavid, D.; Shavel, A.; Alvarez-Puebla, R. A.; Parak, W. J.; Arbiol, J.; Cabot, A. CuTe Nanocrystals: Shape and Size Control, Plasmonic Properties, and Use as SERS Probes and Photothermal Agents. J. Am. Chem. Soc. 2013, 135, 7098-7101.

(26) Wang, S.; Riedinger, A.; Li, H.; Fu, C.; Liu, H.; Li, L.; Liu, T.; Tan, L.; Barthel, M. J.; Pugliese, G.; De Donato, F.; Scotto D’Abbusco, M.; Meng, X.; Manna, L.; Meng, H.; Pellegrino, T. Plasmonic Copper Sulfide Nanocrystals Exhibiting Near-Infrared Photothermal and Photodynamic Therapeutic Effects. ACS Nano 2015, 9, 1788-1800.

(27) Dong, K.; Liu, Z.; Li, Z.; Ren, J.; Qu, X. Hydrophobic Anticancer Drug Delivery by a $980 \mathrm{~nm}$ Laser-Driven Photothermal
Vehicle for Efficient Synergistic Therapy of Cancer Cells in Vivo. Adv. Mater. 2013, 25, 4452-4458.

(28) Liu, Z.; Liu, X.; Du, Y.; Ren, J.; Qu, X. Using Plasmonic Copper Sulfide Nanocrystals as Smart Light-Driven Sterilants. ACS Nano 2015, 9, 10335-10346.

(29) Linic, S.; Aslam, U.; Boerigter, C.; Morabito, M. Photochemical Transformations on Plasmonic Metal Nanoparticles. Nat. Mater. 2015, 14, 567-576.

(30) Lambert, T. N.; Andrews, N. L.; Gerung, H.; Boyle, T. J.; Oliver, J. M.; Wilson, B. S.; Han, S. M. Water-Soluble Germanium(0) Nanocrystals: Cell Recognition and Near-Infrared Photothermal Conversion Properties. Small 2007, 3, 691-699.

(31) Regli, S.; Kelly, J. A.; Shukaliak, A. M.; Veinot, J. G. C. Photothermal Response of Photoluminescent Silicon Nanocrystals. J. Phys. Chem. Lett. 2012, 3, 1793-1797.

(32) Yong, Y.; Cheng, X.; Bao, T.; Zu, M.; Yan, L.; Yin, W.; Ge, C.; Wang, D.; Gu, Z.; Zhao, Y.-l. Tungsten Sulfide Quantum Dots as Multifunctional Nanotheranostics for in Vivo Dual-Modal ImagingGuided Photothermal/Radiotherapy Synergistic Therapy. ACS Nano 2015, 9, 12451-12463.

(33) Yong, Y.; Zhou, L.; Gu, Z.; Yan, L.; Tian, G.; Zheng, X.; Liu, X.; Zhang, X.; Shi, J.; Cong, W.; Yin, W.; Zhao, Y. $\mathrm{WS}_{2}$ Nanosheet as a New Photosensitizer Carrier for Combined Photodynamic and Photothermal Therapy of Cancer Cells. Nanoscale 2014, 6, 1039410403.

(34) Liu, Z.; Liu, J.; Wang, R.; Du, Y.; Ren, J.; Qu, X. An Efficient Nano-Based Theranostic System for Multi-Modal Imaging-Guided Photothermal Sterilization in Gastrointestinal Tract. Biomaterials 2015, $56,206-218$

(35) Liu, J.; Zheng, X.; Yan, L.; Zhou, L.; Tian, G.; Yin, W.; Wang, L.; Liu, Y.; Hu, Z.; Gu, Z.; Chen, C.; Zhao, Y. Bismuth Sulfide Nanorods as a Precision Nanomedicine for in Vivo Multimodal Imaging-Guided Photothermal Therapy of Tumor. ACS Nano 2015, 9, 696-707.

(36) Carrasco, E.; del Rosal, B.; Sanz-Rodríguez, F.; de la Fuente, Á. J.; Gonzalez, P. H.; Rocha, U.; Kumar, K. U.; Jacinto, C.; Solé, J. G.; Jaque, D. Intratumoral Thermal Reading During Photo-Thermal Therapy by Multifunctional Fluorescent Nanoparticles. Adv. Funct. Mater. 2015, 25, 615-626.

(37) del Rosal, B.; Pérez-Delgado, A.; Carrasco, E.; Jovanović, D. J.; Dramićanin, M. D.; Dražić, G.; de la Fuente, Á. J.; Sanz-Rodriguez, F.; Jaque, D. Neodymium-Based Stoichiometric Ultrasmall Nanoparticles for Multifunctional Deep-Tissue Photothermal Therapy. Adv. Opt. Mater. 2016, 4, 782-789.

(38) Othonos, A. Probing Ultrafast Carrier and Phonon Dynamics in Semiconductors. J. Appl. Phys. 1998, 83, 1789-1830.

(39) Giannozzi, P.; Baroni, S.; Bonini, N.; Calandra, M.; Car, R.; Cavazzoni, C.; Ceresoli, D.; Chiarotti, G. L.; Cococcioni, M.; Dabo, I.; Dal Corso, A.; de Gironcoli, S.; Fabris, S.; Fratesi, G.; Gebauer, R.; Gerstmann, U.; Gougoussis, C.; Kokalj, A.; Lazzeri, M.; Martin-Samos, L.; Marzari, N.; Mauri, F.; Mazzarello, R.; Paolini, S.; Pasquarello, A.; Paulatto, L.; Sbraccia, C.; Scandolo, S.; Sclauzero, G.; Seitsonen, A. P.; Smogunov, A.; Umari, P.; Wentzcovitch, R. M. Quantum Espresso: A Modular and Open-Source Software Project for Quantum Simulations of Materials. J. Phys.: Condens. Matter 2009, 21, 395502-395521.

(40) Monkhorst, H. J.; Pack, J. D. Special Points for Brillouin-Zone Integrations. Phys. Rev. B 1976, 13, 5188-5192.

(41) Perdew, J. P.; Burke, K.; Ernzerhof, M. Generalized Gradient Approximation Made Simple. Phys. Rev. Lett. 1996, 77, 3865-3868.

(42) Cococcioni, M.; de Gironcoli, S. Linear Response Approach to the Calculation of the Effective Interaction Parameters in the LDA+ U Method. Phys. Rev. B: Condens. Matter Mater. Phys. 2005, 71, 035105035121.

(43) Roper, D. K.; Ahn, W.; Hoepfner, M. Microscale Heat Transfer Transduced by Surface Plasmon Resonant Gold Nanoparticles. J. Phys. Chem. C 2007, 111, 3636-3641.

(44) Nose, K.; Soma, Y.; Omata, T.; Otsuka-Yao-Matsuo, S. Synthesis of Ternary CuInS 2 Nanocrystals; Phase Determination by Complex Ligand Species. Chem. Mater. 2009, 21, 2607-2613. 
(45) Zhong, H.; Lo, S. S.; Mirkovic, T.; Li, Y.; Ding, Y.; Li, Y.; Scholes, G. D. Noninjection Gram-Scale Synthesis of Monodisperse Pyramidal CuInS 2 Nanocrystals and Their Size-Dependent Properties. ACS Nano 2010, 4, 5253-5262.

(46) Conejeros, S.; Alemany, P.; Llunell, M.; Moreira, I. d. P. R.; Sánchez, V. c.; Llanos, J. Electronic Structure and Magnetic Properties of $\mathrm{CuFeS}_{2}$. Inorg. Chem. 2015, 54, 4840-4849.

(47) Riedinger, A.; Avellini, T.; Curcio, A.; Asti, M.; Xie, Y.; Tu, R. Y.; Marras, S.; Lorenzoni, A.; Rubagotti, S.; Iori, M.; Capponi, P. C.; Versari, A.; Manna, L.; Seregni, E.; Pellegrino, T. Post-Synthesis Incorporation of of ${ }^{64} \mathrm{Cu}$ in $\mathrm{CuS}$ Nanocrystals to Radio Label Photothermal Probes: A Feasible Approach for Clinics. J. Am. Chem. Soc. 2015, 137, 15145-15151.

(48) Tian, Q.; Jiang, F.; Zou, R.; Liu, Q.; Chen, Z.; Zhu, M.; Yang, S.; Wang, J.; Wang, J.; Hu, J. Hydrophilic $\mathrm{Cu}_{9} \mathrm{~S}_{5}$ Nanocrystals: A Photothermal Agent with a $25.7 \%$ Heat Conversion Efficiency for Photothermal Ablation of Cancer Cells in Vivo. ACS Nano 2011, 5, 9761-9771.

(49) Camerin, M.; Rello-Varona, S.; Villanueva, A.; Rodgers, M. A. J.; Jori, G. Metallo-Naphthalocyanines as Photothermal Sensitisers for Experimental Tumours: In Vitro and in Vivo Studies. Lasers Surg. Med. 2009, 41, 665-673.

(50) Protasenko, V.; Bacinello, D.; Kuno, M. Experimental Determination of the Absorption Cross-Section and Molar Extinction Coefficient of CdSe and CdTe Nanowires. J. Phys. Chem. B 2006, 110, 25322-25331.

(51) Mou, J.; Li, P.; Liu, C. B.; Xu, H. X.; Song, L.; Wang, J.; Zhang, K.; Chen, Y.; Shi, J. L.; Chen, H. R. Ultrasmall $\mathrm{Cu}_{2-x} \mathrm{~S}$ Nanodots for Highly Efficient Photoacoustic Imaging-Guided Photothermal Therapy. Small 2015, 11, 2275-2283. 